\title{
Le projet DLCM : gestion du cycle de vie des données de recherche en Suisse
}

Résumé: Dans cet article, les auteurs décrivent le projet national de gestion du cycle de vie des données (DLCM), constitué par les équipes de bibliothèques, informatiques et services de recherche de huit institutions partenaires. Lancé officiellement en août 2015 et financé par le programme swissuniversities SUC P-2/ $\mathrm{P}-5$, le projet vise à mettre en place des services destinés principalement aux chercheurs au niveau suisse pour leurs besoins les plus importants dans la gestion de leurs données. L'identification de ces besoins s'est basée sur une étude d'envergure de documents, ainsi que des entretiens semi-structurés, ce qui a conduit à répertorier des ,use cases‘ principaux ainsi que des services de base, tels que : un point d'accès et de contact pour obtenir des informations, de la formation, et conseils personnalisés, ainsi que des solutions de gestion actives des données, $\mathrm{y}$ inclus des options de stockage de conservation à long terme, et leur publication selon des normes internationales. Un accent particulier a en outre été mis sur la pérénité des services en appliquant des méthodologies de modèles d'affaire inspirés des ,lean startups“ et en établissant des modèles de coûts.

Abstract: Die Autoren beschreiben in diesem Artikel das nationale Projekt Research Data Life-Cycle Management (DLCM), dessen Ziel die Schaffung nachhaltiger und konkreter Lösungen für das Management von Forschungsdaten über den gesamten Lebenszyklus hinweg ist. Das Projekt wurde im August 2015 lanciert und wird über das swissuniversities SUK P-2/P-5 Programm finanziert. Geplant ist, auf nationaler Ebene und für Forschende in der Schweiz Services im Bereich des Forschungsdatenmanagements aufzubauen und so die wichtigsten Bedürfnisse abzudecken. Ausgangspunkt für die Ermittlung dieser Bedürfnisse waren das Studium von Literatur sowie semi-strukturierte Interviews. Auf dieser Basis konnten ,Use Cases ‘ formuliert und Basis-Services definiert werden. Hierzu gehören Kontakt- und Ansprechstellen für Informationen, Weiterbildung, individuelle Beratung, konkrete Lösungen für das Forschungsdatenmanagement, Möglichkeiten der Langzeitarchivierung von Daten sowie deren Publikation nach internationalen Standards. Wichtiger Fokus ist die Nachhaltigkeit dieser Services, wofür auch methodische Ansätze aus dem Bereich der ,Lean Startups` herangezogen und Businessmodelle erarbeitet werden. 


\section{Introduction}

En novembre 2013, Pierre-Yves Burgi a initié des contacts avec des experts du domaine de la gestion des données de recherche dans les hautes écoles suisses. L'intention de cette initiative était d'établir une proposition de projet national qui puisse répondre aux besoins des chercheurs en matière de gestion de leurs données de recherche. Le projet, intitulé DLCM (Data Life-Cycle Management) a débuté officiellement en août 2015, après une période préparatoire initiée en juin 2014. Le projet a été planifié pour une première durée de trois ans avec un financement issu du programme swissuniversities SUC P-2/P-5. Il rassemble des spécialistes de l'information documentaire, des informaticien-ne-s, et chercheuse-r-s de sept hautes écoles, à savoir l'EPFL, la HEG/HES-SO, l'UNIL, l'UNIBAS, l'UZH, l'ETHZ, l'UNIGE ainsi que SWITCH, le fournisseur principal d'infrastructures techniques pour les hautes écoles suisses. Cette collaboration, entre des services de recherche, des départements d'informatique ainsi que des bibliothèques, confère à ce projet un environnement privilégié pour l'échange et le partage d’idées innovatrices en matière de la gestion des données de recherche.

\section{Analyse des besoins}

Afin de mieux connaître les besoins ainsi que les solutions déjà en place dans les institutions partenaires du projet, une analyse exploratoire a été effectuée auprès de chercheur-se-s de différentes disciplines. Au sein de chaque établissement des entretiens semi-structurés ont eu lieu sur une période de deux mois (septembre et octobre 2014). Ces entretiens étaient divisés en quatre parties principales, à savoir :

- données initiales et flux de travail des chercheur-se-s

- analyse et exploration de données

- publication, archivage et gestion de données à long terme

- données de recherche à l'avenir : défis, risques, perspectives

Le tableau 1 présente la compilation de toutes les disciplines impliquées dans les interviews. 
Tab. 1: synthèse des disciplines impliquées dans l'analyse exploratoire

\begin{tabular}{lll}
\hline Institution & $\begin{array}{l}\text { Nombre } \\
\text { d'interviews }\end{array}$ & Disciplines \\
\hline $\begin{array}{l}\text { Université de } \\
\text { Genève (UNIGE) }\end{array}$ & 8 & $\begin{array}{l}\text { Théologie, Informatique, Linguistique, Allemand, } \\
\text { Neurosciences cognitives, Sciences de l'éducation, } \\
\text { Géomatique, Archéologie, Vulnérabilité, Sciences } \\
\text { politiques, Médecine (Recherche sur le cancer de } \\
\text { l'enfant) }\end{array}$ \\
\hline $\begin{array}{lll}\text { ETH Zurich (ETHZ) } \\
\text { Biosystèmes sciences et ingénierie, Réseaux sismiques, } \\
\text { Sociologie, Comportement du consommateur, Groupe } \\
\text { d'optique quantique, Informatique scientifique/Photon } \\
\text { science, Physique }\end{array}$ \\
\hline $\begin{array}{l}\text { Université de } \\
\text { Lausanne (UNIL) }\end{array}$ & 15 & $\begin{array}{l}\text { Médecine sociale, Sciences sociales, Sciences humaines } \\
\text { numériques, Génomique, Biologie des systèmes, } \\
\text { Bioinformatique, Santé publique, Laboratoire d'imagerie } \\
\text { et de médias, Recherche sur le cancer }\end{array}$ \\
\hline $\begin{array}{l}\text { EPF Lausanne } \\
\text { (EPFL) }\end{array}$ & 5 & $\begin{array}{l}\text { Transport et mobilité, Optoélectronique quantique, } \\
\text { Nanomatériaux et interfaces supramoléculaires, } \\
\text { Laboratoire de communication audiovisuelle, Virologie et } \\
\text { génétique }\end{array}$ \\
\hline $\begin{array}{l}\text { Université de Bâle } \\
\text { (UNIBAS) }\end{array}$ & 7 & $\begin{array}{l}\text { Recherche en biologie (Biozentrum, 2), Biologie } \\
\text { (installations de base, 2), Psychologie moléculaire, } \\
\text { Santé publique (STPH), Sciences humaines numériques }\end{array}$ \\
\hline & 5 & $\begin{array}{l}\text { Sciences du droit, Biologie/Microscopie, Biologie/ } \\
\text { Protéomique, Hôpital universitaire, Géosciences }\end{array}$ \\
\hline
\end{tabular}

\section{Données initiales et flux de travail}

Selon cette étude exploratoire, et de manière générale, aucun Data Management Plan (DMP) n'est utilisé, à moins que les bailleurs de fonds l'exigent au moment de la demande de projet, ce qui est par exemple le cas avec le Fonds national suisse (FNS) à partir d'octobre 2017. ${ }^{1}$ En conséquence, la perte de données et le

1 FNS (2017) Open Research Data : les requêtes devront inclure un plan de gestion des données, 6 mars 2017. http://www.snf.ch/fr/pointrecherche/newsroom/Pages/news-170306-open-research -data-bientot-une-realite.aspx. Alle Links in diesem Beitrag wurden am 26.03.2018 überprüft. 
manque de description de ces dernières sont souvent mentionnés comme problématiques.

Un autre défi réside dans l'absence de lignes directrices communes entre les disciplines avec la conséquence que les données existent dans une pluralité de formats (vecteur, vidéo, audio, image, texte, graphique, flux de bits ,bruts‘ etc.). Ces formats sont adaptés aux besoins spécifiques des projets de recherche, et/ou sont imposés par les fabricants des appareils scientifiques, et rarement choisis en vue de la préservation des données, ce qui rend le partage par la suite difficile. Même si des normes de description (métadonnées) communes existent dans certaines disciplines internationalement bien organisées, par exemple en géographie, dans les sciences humaines et les sciences de l'éducation, aucune norme n'est utilisée pour représenter les données, avec parfois même la question de ce que représente précisément une ,donnée‘, ce qui peut paraître surprenant dans certains cas.

En ce qui concerne le stockage des données, dans la plupart des cas, des serveurs autogérés sont utilisés, les services informatiques institutionnels étant souvent peu réactifs pour fournir des solutions à temps. Cependant, indépendamment de la discipline de recherche, les chercheur-se-s sont conscient-e-s de la nécessité de sauvegarder leurs données en plusieurs copies, car la perte de données est une question préoccupante et reconnue. Néanmoins, l'organisation des sauvegardes n'est pas toujours considérée comme une tâche institutionnelle, mais plutôt celle des chercheur-se-s.

\section{Analyse et exploration de données}

Le plus grand défi relevé dans cette partie de l'étude semble être la liberté dans l'organisation des données. Chaque projet de recherche, département, parfois même chaque chercheur-se travaille avec ses propres habitudes ce qui rend l'harmonisation difficile. En ce qui concerne le partage et la conservation, Dropbox (ou solution équivalente) reste une solution utile, même si elle est soumise à la loi américaine sur la vie privée et les droits d'auteur.

\section{Publication, archivage et gestion de données à long terme}

La notion de conservation à long terme est généralement absente. A la question de savoir combien de temps les données devraient être préservées, la réponse ,standard' des chercheur-se-s se situe autour de 10 ans. D’autres questions sans réponses incluent celle concernant la meilleure stratégie pour la conservation à long terme et celles pour savoir s'il existe des règles à ce sujet-là. 
Une autre difficulté dans la conservation est celle liée aux droits d'auteur, car il n’y a pas de compréhension claire sur qui est le propriétaire des données spécifiques ou sur ce qui devrait être entrepris pour publier des données dans le respect de la loi en vigueur.

\section{Les données de recherche à l'avenir : défis, risques, perspectives}

Un point mentionné régulièrement dans cette étude par les chercheur-se-s concerne le manque de réponse adéquate à la question de ce qui pourrait et devrait arriver aux données de recherche après la fin d'un projet et/ou une fois la publication des résultats dans un journal devient effective. Souvent, les données disparaissent dans les tiroirs des bureaux ou sur des serveurs plus ou moins bien entretenus. Bien que la survie de ces données soit préoccupante, leur gestion adéquate est citée comme une question plus difficile.

Les chercheur-se-s interrogé-e-s réitèrent l'importance d'une motivation au partage de données. Une telle incitation leur semble être la citation de données dans la littérature ou de nouvelles méthodes d'évaluation qui valoriseraient le travail de recherche effectué sur les données, comme les data papers. ${ }^{2}$ Une telle valorisation demande cependant à ce que les jeux de données soient identifiés à l'aide d'un lien pérenne, tel que le DOI (Digital Object Identifier). Pour effectuer ce travail de valorisation, une personne complémentaire qui gèrerait les données serait appréciée par les chercheur-se-s interrogé-e-s.

Enfin, les disciplines avec une quantité énorme de données voient également un risque dans la hausse des coûts pour le stockage avec la question sous-jacente de qui va payer pour cette préservation sur le long terme.

\section{Besoins principaux}

Une analyse des principales tendances issues de cette étude exploratoire indique que la gestion des données dépend clairement des stratégies institutionnelles et/ ou des habitudes de recherche dans les disciplines spécifiques. La plupart des

2 E.g. Vishwas Chavan, Lyubomir Penev : The data paper: a mechanism to incentivize data publishing in biodiversity science. In : BMC Bioinformatics 12(Suppl 15)S2 (2011), https://doi.org/ 10.1186/1471-2105-12-S15-S2, as well as Carol Tenopir et al. : Changes in Data Sharing and Data Reuse Practices and Perceptions among Scientists Worldwide. In : PLOS ONE 10(8) e0134826 (2015), https://doi.org/10.1371/journal.pone.0134826. 
disciplines interrogées sont confrontées à différents défis au cours des différentes étapes de leurs projets de recherche. En fait, le cycle de recherche d'aujourd'hui dépend de solutions ad hoc et d'habitudes spécifiques dans des départements de recherche, les besoins principaux suivants ayant été identifiés :

- les lignes directrices et du soutien (institutionnel) pour aider les chercheurs à gérer correctement leurs données

- solutions de stockage, d'informatique et d'analyse de données ad hoc

- solutions pour la gestion active des données, avec stockage des résultats de la recherche basés sur des instantanés périodiques d'ensembles spécifiques de données

- développement et/ou maintenance de dépôts à long terme de données de recherche consultables en ligne

\section{La structure du projet DLCM}

Les résultats de l'enquête des besoins ont permis d'organiser les grands axes du projet DLCM selon cinq sous-projets, dénommés ,tracks', à savoir :

1) lignes directrices et politiques (e.g. DMP)

2) données de recherche actives

3) préservation à long terme

4) conseil, formation et enseignement

5) dissémination/diffusion de l'information sur le plan national et international

Chaque ,track', dirigé par une institution partenaire spécifique, a pour objectif principal de répondre à une partie du cycle de vie des données et de travailler sur des cas d'utilisation concrets. Sur la base de ces derniers, des prototypes sont conçus afin de pouvoir mieux évaluer leur potentialité à devenir des services nationaux.

\section{Lignes directrices et politiques}

Cette partie établit des bonnes pratiques de gestion des données de recherche (dont le DMP), en se basant sur la littérature académique et professionnelle et les meilleures pratiques nationales et internationales. D'autre part un modèle de politique de gestion des données de recherche a été élaboré et présenté au conseil d'administration d'une des plus hautes instances politiques, à savoir la délégation de recherche swissuniversities (dont les membres sont des recteurs/présidents de neuf HEI et les directeurs de la fondation nationale suisse de la science 
et de la Commission de la Technologie et de l'Innovation). L'objectif d'une telle approche est de promouvoir/recommander l'élaboration de politiques basées sur un cadre national commun tout en étant suffisamment souple pour permettre des adaptations pour les spécificités locales.

\section{Données de recherche actives}

Cette étape du cycle de vie des données comprend la collecte, le traitement et l'analyse des données. Sur la base des entretiens des chercheur-se-s, on peut distinguer trois principaux scénarios distincts pour la gestion de ces données actives :

- $\quad$,single endpoint‘, dans lequel soit des données brutes, soit des données qui ont subi plusieurs étapes de traitement sont archivées selon un workflow simple

- $\quad$,open-ended work', qui est représentatif de la gestion active des données qui n'a pas de fin définie et dont les données peuvent évoluer en permanence et/ ou même se référer à d'autres données dans le ,cloud‘ avec une implication évidente sur la notion d'archivage

- data series data', données collectées en continu, éventuellement prétraitées et qui doivent être archivées avant d'être analysées ultérieurement

Ces trois scénarios donnent un aperçu de la complexité de l'articulation entre la gestion active des données et la préservation, car, dans la plupart des cas, il ne s'agit pas d'un processus linéaire, mais plutôt cyclique. Ainsi, cette partie vise à fournir un large éventail de solutions technologiques et des bonnes pratiques pour gérer les données actives en fonction des trois scénarios identifiés, en mettant particulièrement l'accent sur la collecte, le traitement et l'analyse de ces données. Dans cette ligne directrice, trois domaines principaux sont envisagés:

- solutions et support de systèmes de gestion de l'information de laboratoire (Laboratory Information Management System - LIMS) et des cahiers de laboratoire électroniques (Electronic Laboratory Notebook - ELN)

- Virtual Research Environment (VRE) pour les sciences humaines numériques

- une gamme de solutions de travail pour les installations scientifiques et les solutions de stockage de logiciels pour les données actives dans diverses disciplines (avec une attention particulière pour les sciences de la vie et de la santé) 


\section{Préservation à long terme}

Cet axe vise à établir un pont entre les données actives et les solutions de préservation à long terme. Pour ce faire, des concepts bien établis, tels que le Curation Lifecycle $^{3}$ et le modèle OAIS ${ }^{4}$ sont pris en compte. Les principaux livrables de cet axe sont :

- une synthèse des dépôts actuellement utilisés dans les institutions des partenaires du projet ainsi qu'une analyse de leur conformité envers le modèle OAIS

- le développement de la boîte à outils OAIS, avec le paquet d'information à verser (SIP), le paquet d'information archivé (AIP) et le paquet d'information diffusé (DIP)

- la conception et les tests d'un prototype d'une infrastructure évolutive et compatible OAIS

- l'élaboration d'un modèle d'entreprise avec des coûts associés pour conserver les données (de grandes quantités) sur le long terme

\section{Conseil, formation et enseignement}

Pour contrer le manque d'information, voire de formation des chercheur-se-s dans la gestion des données de recherche (RDM), les partenaires du projet DLCM se sont fixés les trois principaux objectifs suivants :

- la création de services de consultation, formation et d'enseignement

- la création d'un service de consultation dans chaque établissement partenaire, coordonné par un bureau central

- l'intégration des connaissances dans les cours Bachelor et Master en Sciences de l'information

\section{Dissémination}

Le projet DLCM, bien qu'il implique de nombreux acteurs suisses dans la gestion des données de recherche au niveau de leurs institutions respectives, a pour ambition de servir plus largement la communauté universitaire suisse. Par consé-

3 Alex Ball : Review of the State of the Art of the Digital Curation of Research Data (version 1.2). Bath 2010.

4 International Organization for Standardisation (2012) ISO 14721:2012: Open archival information system (OAIS), Reference Model. https://www.iso.org/standard/57284.html. 
quent, cet axe vise à promouvoir les résultats du projet DLCM et à établir des contacts et des collaborations pertinents avec d'autres institutions et des projets qui ne sont pas directement impliqués ou liés à celui-ci. Ces activités incluent également le partage de bonnes pratiques avec la communauté internationale lors de participation à des congrès.

\section{Viabilité}

Le projet DLCM étant limité dans le temps et au niveau des ressources, une méthodologie de viabilité basée sur le modèle d'affaire (,business model') a été mise en place pour garantir la durabilité des services à plus long terme. Cette approche, axée sur les client-e-s, se base sur les principes de lean startup développé par Eric Ries ${ }^{5}$, en combinaison avec les outils de référence de l'industrie, à savoir le Business Model Canvas (BMC) et le Value Proposition Canvas (VPC) créés par Alex Osterwalder et Yves Pigneur ${ }^{6}$. Le VPC vise principalement à expliquer comment créer de la valeur pour les client-es, tandis que le BMC explique comment créer de la valeur pour l'entreprise qui fournit le service. Une composante importante de la VPC est de placer les client-e-s au centre et de développer des services en fonction de leurs besoins. Cela devrait en principe éviter de développer des services qui ne seront pas utilisés.

\section{Value Proposition Canvas}

Pour décrire les pratiques des chercheur-se-s, y compris leurs tâches quotidiennes (travail), avec leurs difficultés (en anglais ,pain'), on utilise le VPC (cf. partie droite Fig. 1). Ceci permet d'identifier également les ,gains' potentiels que de nouveaux services pourraient produire. Dans une deuxième étape, la méthode amène à identifier des créateurs de gain (,gain creators') et des remèdes (,pain relievers') et les transformer en produits et services (cf. partie gauche de la Figure 1). De cette façon, chaque service créé répond en principe à un besoin existant et préalablement identifié et offre une valeur spécifique. Un exemple de

5 Eric Ries : The Lean Startup. How Constant Innovation Creates Radically Successful Businesses. London 2011.

6 Alexander Osterwalder, Yves Pigneur : Business Model Generation. A Handbook for Visionaries, Game Changers, and Challengers. Hoboken 2010; Alexander Osterwalder et al. : Value Proposition Design. How to Create Products and Services Customers Want. Hoboken 2014. 
proposition de valeur correspondant aux services liés à la formation est présenté dans la Fig. 1.

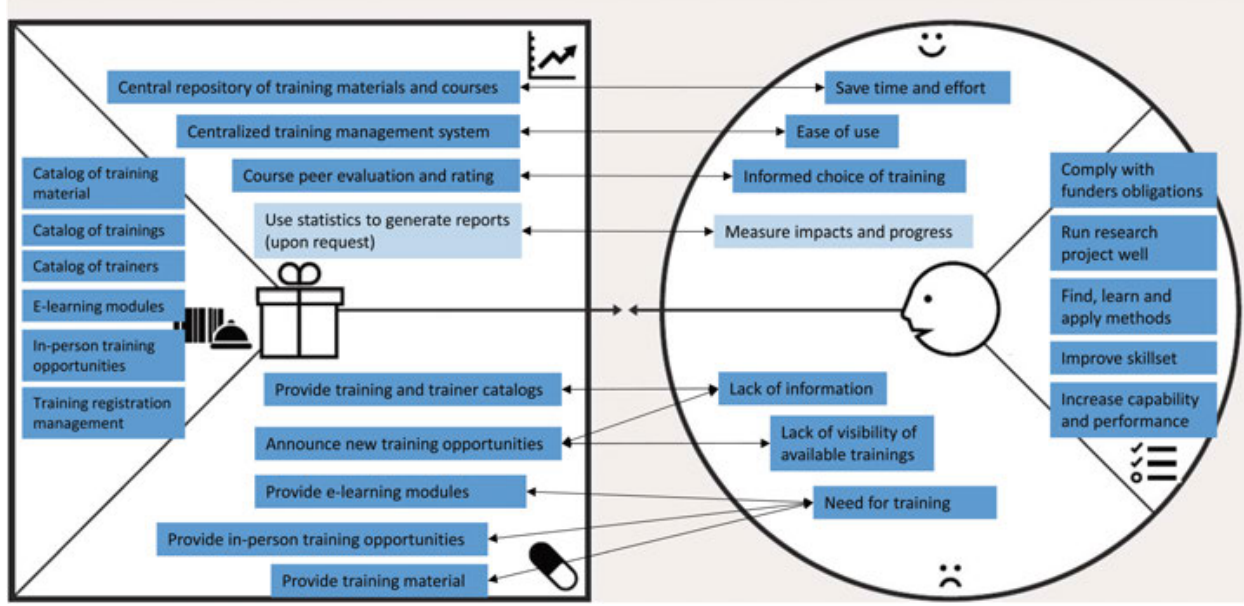

Fig. 1 : Proposition de valeur ${ }^{7}$ (VPC) correspondant aux services de formation pour les chercheurse-s avec à gauche la carte de valeurs (produits et services, créateurs de gain, et remèdes), et à droite (cercle) les gains, les difficultés et les tâches quotidiennes des chercheur-se-s.

\section{Business Model Canvas}

Une fois le VPC élaboré, le BMC est complété en spécifiant d'abord la partie ,Customer Segment' en intégrant le champ ,Value Proposition' préalablement défini, puis en remplissant les autres sections (Customer Relationships, Channels, Revenue Structure, Key Activities, Key Partnernships, Cost Structure) afin de décrire et finaliser les services ciblés. Un exemple de BMC, qui correspond à un service de formation proposé par l'intermédiaire du portail DLCM, dans lequel les chercheur-se-s trouveraient des formations ciblant la gestion de leurs données de recherche, est illustré dans la Fig. 2.

7 Cette proposition de valeurs a été élaborée par Basma Makhlouf-Shabou (HEG / HES-SO) et Lydie Echernier (HES-SO). Merci à elles pour les droits d'utilisation dans ce texte. 


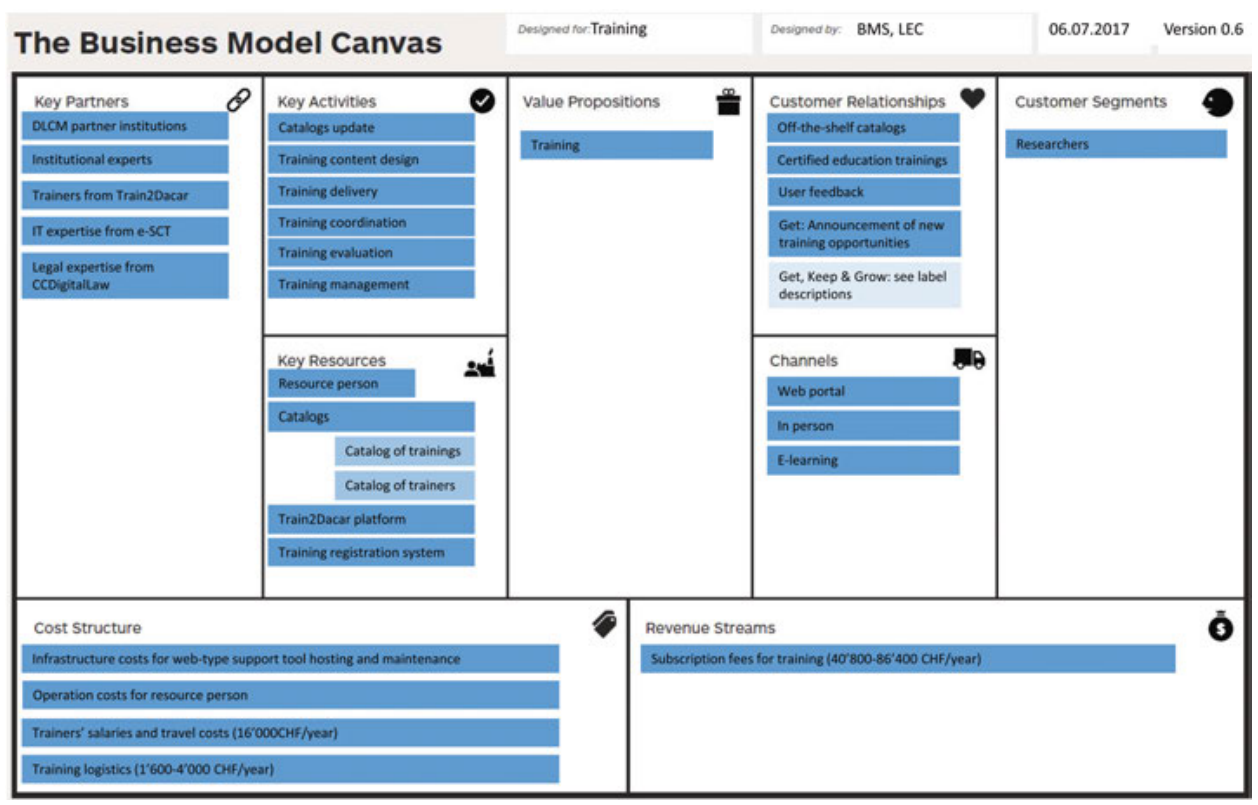

Fig. 2 : Business Model Canvas ${ }^{8}$ (BMC) correspondant au VPC de la Fig. précédente (service de formation destiné aux chercheur-se-s).

\section{Modèles de coûts}

Un composant clé du BMC est sa structure des coûts (voir l'exemple Figure 2), qui devrait dans le cas idéal correspondre aux flux de revenus. Un modèle de coûts particulièrement pertinent et sensible est celui lié à la préservation à long terme. Ce sujet a déjà été abordé dans plusieurs études internationales, notamment le projet KRDS (Keeping Research Data Safe) ${ }^{9}$. Ce projet s'est concentré sur les études de cas du Royaume-Uni pour tirer des conclusions sur les principaux facteurs de coûts, et, sur cette base, a défini un modèle de coûts comprenant les ajustements économiques tels que l'inflation (e.g., le salaire), la déflation (e.g., les supports de stockage), la dépréciation des actifs, et l'amortissement sur investissements. Le modèle prend également en compte les coûts liés au personnel et aux différentes phases du cycle de vie des données (acquisition, ingestion,

8 Ce business model canvas a été élaboré par Basma Makhlouf-Shabou (HEG / HES-SO) et Lydie Echernier (HES-SO). Merci à elles pour les droits d'utilisation dans ce texte.

9 Neil Beagrie et al.: Keeping research safe. A cost model and guidance for UK universities. Final Report, Salisbury 2008. Neil Beagrie et al.: Keeping research safe 2. Final Report. Salisbury 2010. 
archivage, élimination, etc.). Fait intéressant, selon cette étude la sauvegarde et le stockage de fichiers à long terme représentent seulement une petite partie des coûts totaux. L'acquisition et l'ingestion représentent jusqu'à 42\% des coûts, l'accès 35\% et le stockage (,seulement') 23\%.

Outre l'étude KRDS, un autre travail remarquable dans ce domaine est l'étude comparative de plusieurs modèles de coûts réalisés dans le cadre du projet européen $4 \mathrm{C}^{10}$. Un modèle en particulier a retenu notre attention : le TCP (coût total de préservation) du Centre de conservation de l'Université de Californie ${ }^{11}$, qui englobe tous les coûts économiques associés à la préservation à long terme des actifs numériques. Ce modèle prend en compte 11 activités de préservation (système, services, serveurs, personnel, producteurs, flux de travail, types de contenu, stockage, surveillance, interventions, surveillance) et considère deux modèles de prix : ,pay-as-you-go' et ,paid-up“. Dans les deux cas, l'évaluation des 11 activités de préservation est loin d'être triviale et/ou nécessairement fiable, d'autant que cette évaluation se base sur le nombre de client-e-s (qui n'est pas connu à l'avance).

Par conséquent, le modèle de coûts du DLCM se base sur une version simplifiée du TCP, indépendante du nombre de client-e-s, et qui ne tient pas en compte le rendement d'investissement, mais seulement d'un coût global pour le maintien de l'infrastructure, y compris le personnel. D'autres coûts importants liés à des services tels que la création et la curation des données existent, mais pour évaluer les coûts de base, on considère que ces autres services pourraient être facturés séparément en fonction du niveau de services demandé par les client-e-s. Pour les chercheur-se-s avoir des coûts raisonnables pour stocker leurs données à long terme est clairement une incitation importante pour éviter de laisser les données finir leur vie sur une infrastructure de stockage inappropriée (comme les clés USB ou l'ordinateur personnel). Aussi, avec le modèle de coûts DLCM actuel, le prix du TB pour une année pour 3 copies est compris entre CHF 250 et 900 , selon la volumétrie et la durée de préservation. Ces coûts sont alignés sur les prix du marché de façon à rester attractifs pour les chercheur-se-s et institutions de recherche.

\section{Principales réalisations, étapes futures et défis}

Les résultats concrets après deux ans d'activité sont les suivants :

$10 \mathrm{http} / / /$ www.4cproject.eu/.

11 Stephen Abrams et al. : Total Cost of Preservation (TCP). Cost Modeling for Sustainable Services. UC Curation Center, California Digital Library. Los Angeles 2012. 
Lignes directrices, DMP et politiques (track 1) :

Un site Web incluant des ressources, des outils et des lignes directrices pertinents pour les chercheur-se-s a été mis en place (www.dlcm.ch). Un modèle de politique de gestion de données pour guider les institutions dans l'établissement de politiques de gestion de données de recherche a été rédigé et promu auprès de comités directeurs swissuniversities pour la recherche. Enfin, une check-list pour le plan de gestion des données a été élaborée sur la base de l'expérience antérieure de deux institutions partenaires (EPFL et ETHZ). Suite aux exigences récentes (été 2017) du bailleur de fond suisse - le FNS - une collaboration étroite avec ce dernier a été mise sur pied afin d'aligner les besoins et avancements en gestion des données de recherche.

Données de recherche actives (track 2) :

Les principaux axes de cette partie se concentrent sur les environnements LIMS et ELN, un sujet de préoccupation pour les chercheur-se-s disposé-e-s à documenter leurs processus de recherche et leurs données. Une analyse pertinente du marché et des lacunes concernant les logiciels suisses (SLIMS, openBIS, ViKM) et d'autres outils particulièrement utilisés dans les sciences de la vie (par exemple, Labkey) ont été réalisés avec des didacticiels vidéo pour faciliter leur utilisation. Dans une autre étape, comment ces outils peuvent être appliqués à d'autres domaines que les sciences de la vie est en cours d'évaluation (en particulier au département de Physique à l'UNIGE). Pour les humanités digitales (DH), un environnement de recherche virtuel spécifique (SALSAH / KNORA) est en cours d'évaluation en lien avec plusieurs projets en $\mathrm{DH}$.

Préservation à long terme (track 3) :

$\mathrm{Au}$ cours de la première phase du projet, une analyse des écarts utilisant la méthodologie Preserving digital Objects With Restricted Resources (POWRR) ${ }^{12} \mathrm{a}$ été menée afin d'identifier les lacunes pertinentes dans les dépôts institutionnels pour être conformes à la norme OAIS. Le résultat principal de ce travail est l'inventaire des outils et dépôts prévus pour constituer l'écosystème futur du service national de préservation à long terme qui va consister en trois parties : la couche physique de stockage, la couche logique formant le cœur de la solution nationale DLCM qui répond aux spécifications fonctionnelles, et la couche d'interfaces aux utilisateurs (dépôts institutionnels, LIMS/ELN, chercheur-se-s indivi-

12 Jaime Schumacher et al. : From Theory to Action : Good Enough Digital Preservation Solutions for Under-Resourced Cultural Heritage Institutions. A Digital POWRR White Paper for the Institute of Museum and Library Services 2014, doi : http://hdl.handle.net/10843/13610. 
duel-le-s, etc.). La conception de l'architecture orientée ,Web services“ s'est basée sur ces spécifications fonctionnelles afin d'assurer l'interopérabilité selon une approche modulaire pour la construction des paquets d'information SIP, DIP et AIP (Figure 3). Le prototype sera testé avec plusieurs cas d'utilisation de différentes disciplines, des sciences de la vie aux humanités numériques.

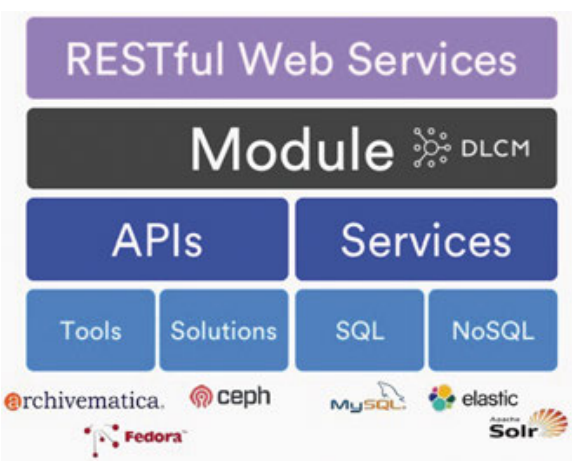

Fig. 3 : Organisation des modules OAIS en services Web. ${ }^{13}$ L'approche modulaire, basée sur les Application Programming Interfaces (API) et des Web services, permet de rendre l'architecture évolutive en fonction des technologies disponibles sur le marché (e.g. archivematica, ceph, Fedora Commons etc.).

Conseil, formation et enseignement (track 4) :

Les premiers ateliers et formations pour la gestion des données de recherche (RDM) destinées aux bibliothécaires ont déjà été dispensés. Parallèlement, un vaste catalogue de modules de formation RDM a été inventorié, y inclus une analyse des besoins pour les étudiants de Bachelor en Sciences de l'information. Un service de consultation générique est en cours de validation dans les hautes écoles de la Suisse occidentale. L'un des résultats attendus consiste en un bureau de coordination centralisé et supporté par un vaste réseau de spécialistes RDM qualifiés représentant leurs institutions universitaires et leurs communautés scientifiques respectives.

Dissemination (track 5) :

A ce stade précoce du projet, l'un des principaux axes de sensibilisation a été d’organiser un événement national annuel sur la thématique RDM ciblant la

13 Ce schéma a été élaboré par Hugues Cazeaux (UNIGE). Merci à lui de nous avoir donné les droits d'utilisation. 
communauté des chercheur-se-s suisses. Le premier ,RDM Day a eu lieu au Rolex Center de l'EPFL en novembre 2016. Il a rassemblé des spécialistes internationaux du domaine RDM et de l'Open Access, ainsi que des représentant-e-s des conseils directeurs. Des ateliers sur de nombreuses thématiques liées aux différents ,tracks' du projet DLCM ont eu lieu en parallèle. Un prochain événement similaire est prévu pour juin 2018 à l’ETHZ.

\section{Etapes futures et défis}

L'ambition de couvrir le cycle de vie complet des données de recherche impose de travailler sur plusieurs fronts en parallèle ce qui complexifie la coordination du projet. Cependant, des résultats commencent à se concrétiser dans chaque ,track', de même une présélection des services potentiels à développer. Cette présélection, basée sur l'analyse BMC et VPC décrite plus haut, devrait faciliter le passage de la phase actuelle de prototypage à celle de la mise en place de services nationaux. Une proposition dans ce sens a été déposée en février 2018 avec l'intention, si la proposition est acceptée, de pouvoir commencer le développement des services nationaux dès août 2018 afin d'avoir une offre de services d'ici 2020. Un défi majeur reste cependant la mise en place de l'organisation nationale qui permettra de gérer les demandes des institutions et chercheur-se-s ainsi que les flux financiers sous-jacents afin d'assurer une viabilité des nouveaux services sur le long terme. 